\title{
Retrospective Analysis of Induction Chemotherapy Plus Concurrent Chemoradiotherapy under Intensity-Modulated Radiotherapy Mode for Locally Advanced Nasopharyngeal Carcinoma
}

Kai Liao

Affiliated Cancer Hospital \& Institute of Guangzhou Medical University

Hao-Yun Tao

Affiliated Cancer Hospital \& Institute Guangzhou Medical University

Ze-Jiang Zhan

Affiliated Cancer Hospital \& Institute Guangzhou Medical University

Wen-Ze Qiu

Affiliated Cancer Hospital \& Institute Guangzhou Medical University

Rong-Hui Zheng ( $\square$ 27819000@qq.com )

Affiliated Cancer Hospital \& Institute Guangzhou Medical University https://orcid.org/0000-0003-40343553

\section{Research Article}

Keywords: Nasopharyngeal carcinoma (NPC), induction chemotherapy, concurrent chemoradiotherapy

Posted Date: June 22nd, 2021

DOI: https://doi.org/10.21203/rs.3.rs-631636/v1

License: (c) (i) This work is licensed under a Creative Commons Attribution 4.0 International License.

Read Full License 


\section{Abstract}

Background: Nasopharyngeal carcinoma (NPC) originates from the mucous epithelium of the nasopharynx. Although induction chemotherapy plus concurrent chemoradiotherapy is the major therapeutic protocol used for locally advanced NPC without metastasis, more researches are needed to evaluate the curative effects.

Aims: We aim to identify the therapeutic effects and prognosis after induction chemotherapy plus concurrent chemoradiotherapy in the treatment of locally advancedNPC under the intensity modulated radiotherapy mode.

Methods: The patients ( $\mathrm{N}=544)$ with locally advanced NPC (III and IVa) after intensity modulated radiotherapy with induction chemotherapy and concurrent chemoradiotherapy were included in this study. We analyzed the characteristics of patients including gender, age, smoking status, tumor-node staging system, clinical stage, pathological type, the therapy protocol of induction chemotherapy and concurrent chemoradiotherapy, and chemotherapy prescription.

Results: We have found the 5-year survival rates of OS, PFS, LRRFS, and DMFS were $85.21 \%, 78.51 \%$, $90.71 \%$, and $85.21 \%$ in follow-up and these data indicated that our therapeutic procedure provided beneficial effects on survival rates. Following, the chemotherapy drug base on DOC provided more beneficial effect of survival rate compared with TXT but there was no significant difference between chemotherapy drug base on DDP and NDP treated with NPC patients. The therapeutic effects of induction chemotherapy revealed no difference between TPF and TP. In addition, there was also no significant change between concurrent chemotherapy with TP dual drugs or platinum single drug. Moreover, the survival rate showed no difference between platinum accumulation dose ofmore and less than $150 \mathrm{mg} / \mathrm{m} 2$ for concurrent chemotherapy.

Conclusion: Our results indicate that induction chemotherapy plus concurrent chemoradiotherapy under intensity modulated radiotherapy which is the standard therapeutic method for locally advanced NPC provides beneficial therapeutic effects, and it is worthy of further study.

\section{Introduction}

Nasopharyngeal carcinoma (NPC) originates from the mucous epithelium of the nasopharynx and is the most commonly diagnosed malignant head and neck tumor,which accounts for approximately $60 \%$ of all head and neck tumors (1). The characteristics of NPC incidence have been observed with regional, ethnic, and familial epidemiological. Moreover, the incidence of NPC shows the difference from region to region and is prevalent in East Asia, Southeast Asia, East Africa, and North Africa. For now, China accounts for approximately $47.7 \%$ of all new cases of NPC worldwide(2). Accordingly, the incidence of NPC in China also showssignificant regional differences. In South China, it has been reported the highest prevalence of Hunan, Guangdong, Guangxi, and Fujian (3). Because of the special location andhighly sensitive response to radiotherapy, surgery is not an appropriate therapeutic method and radiotherapy is the only 
curative treatment for non-metastatic NPC $(4,5)$. Nowadays, staging system and target volume delineation base on magnetic resonance imaging (MRI) ,and treatment with intensity modulated radiotherapy (IMRT) technique, are standard mode for the NPC patients(6).

On clinical, radical radiotherapy is a standard therapeutic method for the early stage of NPC to provide the best therapeutic effect. However, the special location of NPC provides the occult nature and leads to diagnostic difficulty. Accordingly, over $70 \%$ of NPC patients are diagnosed as locally advanced stage(7).

According to the report published in 1998, therapeutic protocol using platinum drugs for concurrent chemoradiotherapy plus three-cycle platinum drugs plus 5-FU (PF) as the adjuvant chemotherapy for locally advanced NPC has been used for more than 10 years. Nowadays, concurrent chemoradiotherapy which is the major therapeutic method for NPC is still the footstone,but the utilization rate ofadjuvant chemotherapy is decreasing and induction chemotherapyis gradually increasing. The clinical trial report from Sun Yat-sen University (5) indicated that compared with the concurrent chemoradiotherapy group, the 3-cycle PF regimen adjuvant chemotherapy failed to further improve the patient's prognosis, but increased adverse reactions. Accordingly, induction chemotherapy before radiotherapy will decrease the metastasis rate of NPC patientsand providemore therapeutic tolerance and sensitivity compared with adjuvant chemotherapy (8). Importantly, the research from Sun Yat-sen University using induction chemotherapy instead of adjuvant chemotherapy showed the results that 3-cycle with TPF or GP of induction chemotherapy plus concurrent chemoradiotherapy significantly increased PFS and OS in locally advanced NPC patient compared to the patient who treated with concurrent chemoradiotherapy only $(9,10)$. Taken together, induction chemotherapy plus concurrent chemoradiotherapy is an important therapeutic protocol for patients with locally advanced NPC but still needs more evidence to confirm the therapeutic effects.

Therefore, this study conducted a retrospective analysis of induction chemotherapy plus concurrent chemoradiotherapy in the treatment of locally advanced NPCunder the intensity modulated radiotherapy mode to further explore their therapeutic effects and prognosis.

\section{Methods}

\section{Study patients}

In this study, we evaluated the patients with locally advanced NPC (III and IVa,UICC 8th) after intensity modulated radiotherapy with induction chemotherapy and concurrent chemoradiotherapy.Finally, 544 NPC patients were included between January 2009 and December 2015 from "Affiliated Cancer Hospital \& Institute of Guangzhou Medical University" who had already finished induction chemotherapy with concurrent chemoradiotherapy and without adjuvant chemotherapy. Following, the TP or TPF (T: docetaxel(DOC) or Taxol(TXT), P: cisplatin(DDP) or nedaplatin(NDP),F: 5-fluorouracil) was the treatment protocol for induction chemotherapy, with platinum drug (cisplatin or nedaplatin) or TP used for 
concurrent chemotherapy. Moreover, each patient was treated with the same chemodrug during the course of induction chemotherapy and concurrent chemoradiotherapy. This study was approved by the

Research Ethics Committee of the Affiliated Cancer Hospital \& Institute of Guangzhou Medical University. The requirement of patient informed consent was waived in view of the retrospective nature of the study.

\section{Induction chemotherapy(IC)}

An IC regimen with TPF or TP was used in this study. The TPF regimen included docetaxel/taxol (60 $\mathrm{mg} / \mathrm{m} 2$, d1/135 mg/m2,d1), DDP/NDP (60 mg/m2, d1/60 mg/m2, d1) and 5-fluorouracil $(600 \mathrm{mg} / \mathrm{m} 2,24$ hours daily from d1 $\sim 5)$. The TP regimen included docetaxel/taxol $(75 \sim 80 \mathrm{mg} / \mathrm{m} 2$, d1/135 175 $\mathrm{mg} / \mathrm{m} 2)$ and DDP /NDP (75 $80 \mathrm{mg} / \mathrm{m} 2, \mathrm{~d} 1 / 75 \sim 80 \mathrm{mg} / \mathrm{m} 2, \mathrm{~d} 1)$. IC was administered every three weeks, with $2 \sim 3$ cycles.

\section{Concurrent chemoradiotherapy (CCRT)}

Radiotherapy is the main treatment for NPC. All patients were irradiated with IMRT. After being immobilized with head and neck thermoplastic masks while in the supine position, patients were scanned with a CT simulator. Noncontrast and contrast CT

images were collected with $3 \mathrm{~mm}$ per slice from the vertex to $2 \mathrm{~cm}$ below the clavicle head. The target volumes were contoured according to the Sun Yat-sen University Cancer Center institutional treatment protocol $(5,11)$, which is in agreement with the International Commission on Radiation Units and Measurements Reports 50 and 62 . The prescribed radiation dose to the gross tumor volume of nasopharyngeal tumors (GTVnx) was $70 \mathrm{~Gy}$, and the prescribed radiation dose to the gross tumor volume of lymph nodes (GTVnd) was $68 \mathrm{~Gy}$. The high-risk clinical target volume (CTV1) and the low-risk clinical target volume (CTV2) were prescribed $62 \mathrm{~Gy}$ and $56 \mathrm{~Gy}$, respectively. All patients were irradiated with 32 to 33 fractions in total, once daily, Monday through Friday. Patients were treated with $1 \sim 3$ cycles of DDP /NDP(75 $100 \mathrm{mg} / \mathrm{m}^{2}, \mathrm{~d} 1 / 75 \sim 100 \mathrm{mg} / \mathrm{m}^{2}$, d1) every 21 days during radiotherapy,or were given DDP/NDP $\left(75 \sim 80 \mathrm{mg} / \mathrm{m}^{2}, \mathrm{~d} 1 / 75 \sim 80 \mathrm{mg} / \mathrm{m}^{2}, \mathrm{~d} 1\right)$ and Docetaxel/taxol $(75 \sim 80 \mathrm{mg} / \mathrm{m} 2, \mathrm{~d} 1 / 135 \sim 175$ $\mathrm{mg} / \mathrm{m} 2 \mathrm{~d} 1$ ) which was repeated every 21 days for $1 \sim 3$ cycles during radiotherapy.

\section{Follow-up}

After treatment, the patients were followed every 3 months for 2 years, every 6 months from the 3rd to 5th years, and every year thereafter. Follow-up data were obtained from outpatient and inpatient medical records and telephone counseling. OS was calculated from the date of diagnosis until the follow-up deadline or death. PFS was defined as the time from initial diagnosis to tumor progression or death; DMFS was defined as the time to tumor metastasis, and locoregional relapse-free survival (LRRFS) was defined as the time to the first locoregional recurrence. Hematologic toxicity, liver and kidney function, and oral mucosal responses were graded according to the National Cancer Institute Common Terminology Criteria for Adverse Events Version 4.0 (NCI-CTCAE 4.0)

\section{Statistical analysis}


There were four major endpoints in this study, including OS $\square$ PFS $\square$ LRRFS, and DMFS. Continuous data were indicated with mean \pm standard deviation (SD) while categorical data were indicated with number and percentage (\%). Univariate Cox proportional-hazard regression was used for stratified analysis of all independent variables. Further, a multivariate Cox proportional-hazard regression with forward(Wald) procedure was used to investigate the associated factors to OS, PFS, LRRFS, and DMFS. The variables which were significant in univariate Cox regression results would be entered into the multivariate model with the following six variables: clinical stage, induction chemotherapy, concurrent chemotherapy, CT drug base on DOC/TXT, CT drug base on DDP/NDP, platinum dose of CCRT. Then, the variables which were significant in the multivariate model would be recognized as associated factors to OS, PFS, LRRFS, or DMFS. Kaplan-Meier survival analysis and log-rank test were used to compare the survival trends of categorical variables. A P $<0.05$ would be recognized as reaching significance of each test, two-tailed. All analyses were performed using IBM SPSS Version 25 (SPSS Statistics V25, IBM Corporation, Somers, New York).

\section{Results}

\section{Patient's clinical characteristics}

We included 544 patients in this study and the patients' clinical characteristics were indicated in Table 1. The averaging age and BMI of patients were $46.09 \pm 11.16$ years and $22.71 \pm 3.29 \mathrm{~kg} / \mathrm{m}^{2}$, the gender ratio was 2.60:1 (male/female=393/151), $41.91 \%$ of patients smoked. The major pathology type was undifferentiated, nonkeratinizing squamous cell carcinoma (97.61\%). The clinical stages of patients were $64.52 \%$ in stage 3 and $35.48 \%$ in stage 4 a. The methods of induction chemotherapy were $418(76.84 \%)$ patients with TP and $126(23.16 \%)$ patients with TPF, and of concurrent chemotherapy were 167 (30.70\%) patients with TP and 377 (69.30\%) patients with P. The drugs of induction and concurrentChemotherapy were 504 (92.65\%) patients with DOC and 40 (7.35\%) patients with TXT, and were $142(26.10 \%)$ patients with DDP and 402 (73.90\%) patients with NDP. There were $53.49 \%$ of patients received $\geq 150 \mathrm{mg} / \mathrm{m}^{2}$ of platinum dose of CCRT while the other $46.51 \%$ was $<150 \mathrm{mg} / \mathrm{m}^{2}$.

\section{Clinical outcomes}

\section{Overall survival results}

The median follow-up time was 64 months (4 114 months). There were 112 (20.59\%) PFS, 83 (15.26\%) death, 45 (8.27\%) LRRFS, and 76 (13.97\%) DMFS being observed in follow-up. Figure 1 shows the overall survival results of all patients in OS, PFS, LRRFS, and DMFS. The 5-year estimated survival rates of OS, PFS, LRRFS, and DMFS were $85.21 \%, 78.51 \%, 90.71 \%$, and $85.21 \%$, respectively. Figure 2 further illustrates the survival trends by clinical stages. All log-rank test results were significant between stage 3 and $4 a$ (P from 0.039 to $<0.001)$. The 5 -year estimated survival rates in stage 3 of OS, PFS, LRRFS, and 
DMFS were 90.49\%, 84.71\%, 92.30\%, and 90.94\%, respectively. And the 5-year estimated survival rates in stage $4 \mathrm{a}$ of OS, PFS, LRRFS, and DMFS were $76.01 \%, 67.49 \%, 87.84 \%$, and $74.91 \%$, respectively.

\section{Stratified analysis of OS, PFS, LRRFS, and DMFS}

Table 2 shows the univariately stratified analyses of all endpoints by all independent variables. The significant variables which were associated with higher risk of each endpoint were listed as follows. For OS, higher age, $\mathrm{N}$ stage, clinical stage, lower platinum dose of CCRT, and concurrent CT of P. For PFS, higher BMI, N stage, clinical stage, and concurrent CT of P. For LRRFS, smoking and higher clinical stage. For DMFS, higher BMI, N stage, clinical stage, concurrent CT of P, and CT drug base on TXT.

\section{Associated factors to OS, PFS, LRRFS, and DMFS}

To further investigate the associated factors to endpoints, multivariate Cox proportional-hazard regression was carried out and results are listed in Table3. As indicated, Age over 46 was associated with the risk of OS $(P=0.024)$. Higher $B M I$ was associated with the risk of DMFS $(P=0.033)$. Higher $N$ stage was associated with the risk of OS, PFS, and DMFS (all $\mathrm{P}<0.05$ ). Clinical stage was still associated with all endpoints (all P<0.05). CT drug base on TXT had higher risk of OS, PFS, and DMFS (all P<0.05).

\section{Accute side effects}

The CTC4.0 levels of hematological results, radiation-induced oral mucositis, gastrointestinal reaction, liver function, and renal function during IC+CCRT are listed in Table 4. Most patients were at level 1-2 in hematology results (59.50\%), radiation-induced oral mucositis (73.85\%), and gastrointestinal reaction (88.21\%). Most patients were at level 0 in liver function (62.35\%), and renal function (88.49\%).

\section{Discussion}

In summary, we obtained that the 5 years survival rates of OS, PFS, LRRFS, and DMFS were 85.21\%, $78.51 \%, 90.71 \%$, and $85.21 \%$,showing beneficial effects after induction chemotherapy plus concurrent chemoradiotherapy on clinical patients with locally advanced NPC. Moreover, the previous study indicated that the NPC patients who treated with induction chemotherapy with TPF plus concurrent chemoradiotherapy showed the 5 years OS (85.6\%), PFS (77.4\%), LRRFS rate (90.7\%) and DMFS (88\%) (12), which were similar to our results.

Mainly, there are four therapeutic protocols of induction chemotherapy formulary such as TP, TPF, GP, and PF for locally advanced NPC. However, there is no prospective clinical trial evidence to compare the difference between the four therapeutic protocols. Following, TPF and GP are the NCCN Guidelines Class $1 \mathrm{~A}$ recommendation, but considering the higher hematological toxicity of TPF and GP,TP with less toxicicy is also commonly used clinically. We discovered that there was no significant difference in OS, 
PFS, LRRFS, and DMFS between TPF and TP formularies. Following, these results show that TP for induction chemotherapy on locally advanced NPC patients may be enough but we still need more prospective research to provide evidence to confirm this result. Moreover, the previous study indicated that cancer patients treated with DDP usually lead to strong side effects such as severe kidney injury, acute allergic reactions, decreased immune response, gastrointestinal injury, hemorrhage reactions. Patients treated with NDP will also lead to several side effects but less than DDP (13). Following, our data showed that the survival rate was no significant difference between locally advanced NPC patients who were treated with DDP or NDP. Taken together, the above information suggests that choosing NDP as the chemodrug used for NPC patients will not change the curative effect and may decrease the side effect (14). Moreover, we also compared NPC patients who were treated with TP or P for concurrent chemoradiotherapy and showed the survival rate was no difference between these two groups. Accordingly, in locally advanced lung cancerand esophageal cancer, the concurrent chemoradiotherapy regimen is the use of double-drug regimen containing platinum $(15,16)$. Recently, a retrospective analysis obtained that the treament with double-drug regimen containing platinum for concurrent chemotherapy for NPC was better than single platinum drug but the side effects increased significantly(17). Our results suggests that under the premise of induction chemotherapy, concurrent chemotherapy of single platinum drug for NPC may be sufficient but need more studies to confirm.

Accordingly, paclitaxel drugs such as DOC and TXT are the first-line chemodrug used on clinical patients (13). Moreover, the previous studies indicated that the mechanism of action of DOC was through decreased Bcl-2 expression and lead to mitochondria-dependent apoptosis but TXT was regulation of the microtubules stabilization to arrest cell cycle $(18,19)$. Our study indicated that patients treated with DOC could extend the survival rate compared with patientstreated with TXT. These findings may provide the possibility that DOC is more suitable for chemotherapy of NPC. In addition, previous research found that more than $200 \mathrm{mg} / \mathrm{m}^{2}$ of platinum accumulated dosage during CCRT could extend the survival rate (20) base on concurrent chemoradiotherapy only without induction chemotherapy. Accordingly, the patients treated with induction chemotherapy plus concurrent chemoradiotherapy whether can decreased the accumulation of platinum dosage, needing more pieces of evidence. Moreover, other research had shown that under the premise of induction chemotherapy, more or less than $160 \mathrm{mg} / \mathrm{m}^{2}$ of accumulation of platinum dose during CCRT period was no difference in survival rate, suggesting that the accumulation of platinum dosage can be reduced to $160 \mathrm{mg} / \mathrm{m}^{2}(21)$. In this study, we obtained that the survival rate of patients did not significantly change between platinum dose of over $150 \mathrm{mg} / \mathrm{m}^{2}$ and less than 150 $\mathrm{mg} / \mathrm{m}^{2}$. These messages indicate that the accumulation of platinum doseduring CCRT under the premise of induction chemotherapy is worthy of further research.

Although our study provides the beneficial effects of concurrent chemoradiotherapy plus induction chemotherapy under intensity modulated radiotherapy to treat locally advanced NPC patients,there are still some limitations of this study. In retrospective studies, it is difficult to avoid that potential prognosticrelated factors are not included and cause deviations such as EBV-DNA,because our center was unable to 
perform this test before 2015. Moreover, because of the incomplete data records of the medical records, the long-term toxicity after radiotherapy could not be counted and reported.

This study confirms that induction chemotherapy plus concurrent chemoradiotherapy which is the standard therapeutic mode for locally advanced nasopharyngeal carcinoma shows beneficial efficacy and safety ,and it is worthy of further research.

\section{Declarations}

\section{Funding}

None declared.

\section{Conflicts of interest}

There are no conflicts of interest of the authors.

\section{Availability of data and material}

All data generated or analyzed during this study are included in this published article.

\section{Code availability}

Not applicable.

\section{Author Contributions}

Kai Liao and Rong-Hui Zheng conceived and designed the project. Kai Liao, Hao-Yun Tao and Ze-Jiang Zhan collected the data. Kai Liao, Hao-Yun Tao and Wen-ze Qiu analyzed and interpreted the data. RongHui Zheng and Kai Liao drafted the manuscript. All authors read and approved the final manuscript.

Acknowledgments: We sincerely express our gratitude to Affiliated Cancer Hospital \& Institute of Guangzhou Medical University and patients in this study.

\section{References}

1. Cao SM, Simons MJ, Qian CN. The prevalence and prevention of nasopharyngeal carcinoma in China. Chin J Cancer. 2011;30(2):114-9.

2. Chen YP, Chan ATC, Le QT, Blanchard P, Sun Y, Ma J. Nasopharyngeal carcinoma. Vol. 394, The Lancet. 2019. p. 64-80.

3. Wei WI, Sham JST. Nasopharyngeal carcinoma. In: Lancet. 2005. p. 2041-54.

4. Baujat B, Audry H, Bourhis J, Chan ATC, Onat H, Chua DTT, et al. Chemotherapy in locally advanced nasopharyngeal carcinoma: An individual patient data meta-analysis of eight randomized trials and 1753 patients. In: International Journal of Radiation Oncology Biology Physics. 2006. p. 47-56. 
5. Chen L, Hu CS, Chen XZ, Hu GQ, Cheng Z, Bin, Sun Y, et al. Concurrent chemoradiotherapy plus adjuvant chemotherapy versus concurrent chemoradiotherapy alone in patients with locoregionally advanced nasopharyngeal carcinoma: A phase 3 multicentre randomised controlled trial. Lancet Oncol. 2012;13(2):163-71.

6. Kwong DLW, Pow EHN, Sham JST, McMillan AS, Leung LHT, Leung WK, et al. Intensity-modulated radiotherapy for early-stage nasopharyngeal carcinoma: A prospective study on disease control and preservation of salivary function. Cancer. 2004;101(7):1584-93.

7. Mao YP, Xie FY, Liu LZ, Sun Y, Li L, Tang LL, et al. Re-Evaluation of 6th Edition of AJCC Staging System for Nasopharyngeal Carcinoma and Proposed Improvement Based on Magnetic Resonance Imaging. Int J Radiat Oncol Biol Phys. 2009;73(5):1326-34.

8. Guo L, Lin HX, Xu M, Chen QY, Wang CT, Huang PY. Phase I study of TPF neoadjuvant chemotherapy followed by radical radiotherapy in advanced nasopharyngeal carcinoma. Chin J Cancer. 2010;29(2):136-9.

9. Sun Y, Li WF, Chen NY, Zhang N, Hu GQ, Xie FY, et al. Induction chemotherapy plus concurrent chemoradiotherapy versus concurrent chemoradiotherapy alone in locoregionally advanced nasopharyngeal carcinoma: a phase 3, multicentre, randomised controlled trial. Lancet Oncol. 2016;17(11):1509-20.

10. Zhang Y, Chen L, Hu G-Q, Zhang N, Zhu X-D, Yang K-Y, et al. Gemcitabine and Cisplatin Induction Chemotherapy in Nasopharyngeal Carcinoma. N Engl J Med. 2019;381(12):1124-35.

11. Zhao C, Han F, Lu LX, Huang SM, Lin CG, Deng XW, et al. Intensity modulated radiotherapy for localregional advanced nasopharyngeal carcinoma. Ai Zheng. 2004;23(11 Suppl):1532-7.

12. Li WF, Chen NY, Zhang N, Hu GQ, Xie FY, Sun Y, et al. Concurrent chemoradiotherapy with/without induction chemotherapy in locoregionally advanced nasopharyngeal carcinoma: Long-term results of phase 3 randomized controlled trial. Int J Cancer. 2019;145(1):295-305.

13. Lin S, Lu JJ, Han L, Chen Q, Pan J. Sequential chemotherapy and intensity-modulated radiation therapy in the management of locoregionally advanced nasopharyngeal carcinoma: Experience of 370 consecutive cases. BMC Cancer. 2010;10:39.

14. Tang LQ, Chen DP, Guo L, Mo HY, Huang Y, Guo SS, et al. Concurrent chemoradiotherapy with nedaplatin versus cisplatin in stage II-IVB nasopharyngeal carcinoma: an open-label, non-inferiority, randomised phase 3 trial. Lancet Oncol. 2018;19(4):461-73.

15. Paz-Ares L, Dvorkin M, Chen Y, Reinmuth N, Hotta K, Trukhin D, et al. Durvalumab plus platinumetoposide versus platinum-etoposide in first-line treatment of extensive-stage small-cell lung cancer (CASPIAN): a randomised, controlled, open-label, phase 3 trial. Lancet. 2019;394(10212):1929-39.

16. Wang YS, Tian J, Han Y, Han SM, Shi S, Bin. Gemcitabine plus vinorelbine as second-line therapy in patients with metastatic esophageal cancer previously treated with platinum-based chemotherapy. Oncol Res. 2016;24(2):129-35.

17. Tao HY, Zhan ZJ, Qiu WZ, Liao K, Yuan YW, Yuan TZ, et al. Clinical value of docetaxel plus cisplatin (TP) induction chemotherapy followed by TP concurrent chemoradiotherapy in locoregionally 
advanced nasopharyngeal carcinoma. J Cancer. 2021;12(1):18-27.

18. Wongsaengsak S, Quirch M, Ball S, Sultan A, Jahan N, Elmassry M, et al. Docetaxel-induced acute myositis: a case report with review of literature. J Chemother. 2021;33(2):116-21.

19. Xu J, Feng Z, Chen S, Zhu J, Wu X, Chen X, et al. Taxol alleviates collagen-induced arthritis in mice by inhibiting the formation of microvessels. Clin Rheumatol. 2019;38(1):19-27.

20. Zhang Y, Zheng J, Jiang Y, Huang X, Fang L. Neglected, Drug-Induced Platinum Accumulation Causes Immune Toxicity. Front Pharmacol. 2020;11:1166.

21. Lv JW, Qi ZY, Zhou GQ, He XJ, Chen YP, Mao YP, et al. Optimal cumulative cisplatin dose in nasopharyngeal carcinoma patients receiving additional induction chemotherapy. Cancer Sci. 2018;109(3):751-63.

\section{Tables}

\section{Table 1. Patient's clinical characteristics}




\begin{tabular}{|c|c|}
\hline Parameters & All $(n=544)$ \\
\hline \multicolumn{2}{|l|}{ Sex } \\
\hline Male & $393(72.24 \%)$ \\
\hline Female & $151(27.76 \%)$ \\
\hline Age, year & $46.09 \pm 11.16$ \\
\hline$<46$ & $261(47.98 \%)$ \\
\hline$\geq 46$ & $283(52.02 \%)$ \\
\hline $\mathrm{BMI}, \mathrm{kg} / \mathrm{m} 2$ & $22.71 \pm 3.29$ \\
\hline$<18$ & $24(4.41 \%)$ \\
\hline $18-24$ & $350(64.34 \%)$ \\
\hline$>24$ & $170(31.25 \%)$ \\
\hline \multicolumn{2}{|l|}{ Smoking } \\
\hline No & $316(58.09 \%)$ \\
\hline Yes & $228(41.91 \%)$ \\
\hline \multicolumn{2}{|l|}{ Pathology } \\
\hline Keratoacanthoma & $3(0.55 \%)$ \\
\hline Nonkeratinized squamous cell carcinoma & $10(1.84 \%)$ \\
\hline Undifferentiated, nonkeratinizing squamous cell carcinoma & $531(97.61 \%)$ \\
\hline \multicolumn{2}{|l|}{ T stage } \\
\hline 1 & $18(3.31 \%)$ \\
\hline 2 & 107 (19.67\%) \\
\hline 3 & 291 (53.49\%) \\
\hline 4 & $128(23.53 \%)$ \\
\hline \multicolumn{2}{|l|}{$\mathrm{N}$ stage } \\
\hline 0 & $31(5.70 \%)$ \\
\hline 1 & $128(23.53 \%)$ \\
\hline 2 & 294 (54.04\%) \\
\hline 3 & 91 (16.73\%) \\
\hline
\end{tabular}




\begin{tabular}{ll}
3 & $351(64.52 \%)$ \\
\hline $4 a$ & $193(35.48 \%)$ \\
\hline Induction chemotherapy & $418(76.84 \%)$ \\
\hline TP & $126(23.16 \%)$ \\
\hline TPF & \\
\hline Concurrent chemotherapy & $167(30.70 \%)$ \\
\hline TP & $377(69.30 \%)$ \\
\hline P & \\
\hline CT drug base on DOC/TXT & $504(92.65 \%)$ \\
\hline DOC & $40(7.35 \%)$ \\
\hline TXT & \\
\hline CT drug base on DDP/NDP & $142(26.10 \%)$ \\
\hline DDP & $402(73.90 \%)$ \\
\hline NDP & $147.70 \pm 51.27$ \\
\hline Platinum dose of CCRT, mg/m2 & $253(46.51 \%)$ \\
\hline$<150$ & $291(53.49 \%)$ \\
$\geq 150$ &
\end{tabular}

Table 2. Stratified analysis of independent variables to OS, PFS, LRRFS, and DMFS 
OS PFS LRRFS DMFS

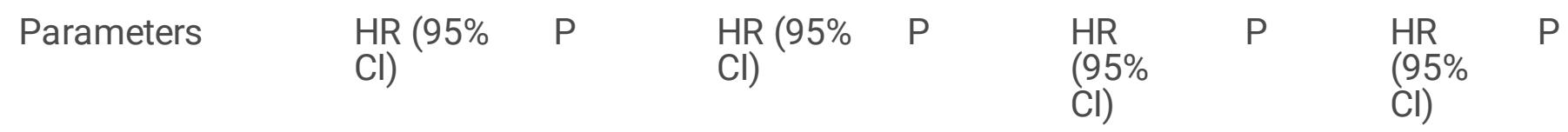

Sex

$\begin{array}{lllllllll}\text { Male } & \text { ref. } & - & \text { ref. } & - & \text { ref. } & - & \text { ref. } & - \\ \text { Female } & 0.87 & 0.588 & 0.93 & 0.730 & 1.04 & 0.903 & 0.84 & 0.518 \\ & (0.53 \text { to } & & (0.61 \text { to } & & (0.55 & & (0.50 & \text { to } \\ & 1.43) & & 1.41) & & \text { to } & 1.98) & & 1.42)\end{array}$

Age, year

$\begin{array}{lllllllll}<46 & \text { ref. } & - & \text { ref. } & - & \text { ref. } & - & \text { ref. } & - \\ \geq 46 & 1.57 & 0.046 & 1.27 & 0.211 & 1.45 & 0.221 & 1.17 & 0.503 \\ & (1.01 \text { to } & & (0.87 \text { to } & & (0.80 & \text { to } & (0.74 & \text { to } \\ & 2.44) & & 1.85) & & 2.63) & & 1.83)\end{array}$

\begin{tabular}{|c|c|c|c|c|c|c|c|c|}
\hline $\mathrm{BMI}, \mathrm{kg} / \mathrm{m} 2$ & & 0.050 & & 0.041 & & 0.755 & & 0.020 \\
\hline$<18$ & ref. & - & ref. & - & ref. & - & ref. & - \\
\hline $18-24$ & $\begin{array}{l}1.02 \\
(0.41 \text { to } \\
2.56)\end{array}$ & 0.958 & $\begin{array}{l}1.01 \\
(0.44 \text { to } \\
2.31)\end{array}$ & 0.984 & $\begin{array}{l}0.71 \\
(0.22 \\
\text { to } \\
2.34)\end{array}$ & 0.577 & $\begin{array}{l}1.08 \\
(0.39 \\
\text { to } \\
2.98)\end{array}$ & 0.877 \\
\hline$>24$ & $\begin{array}{l}0.52 \\
(0.19 \text { to } \\
1.42)\end{array}$ & 0.202 & $\begin{array}{l}0.56 \\
(0.23 \text { to } \\
1.38)\end{array}$ & 0.209 & $\begin{array}{l}0.62 \\
(0.18 \\
\text { to } \\
2.19)\end{array}$ & 0.462 & $\begin{array}{l}0.46 \\
(0.15 \\
\text { to } \\
1.41)\end{array}$ & 0.174 \\
\hline
\end{tabular}

Smoking

$\begin{array}{lllllllll}\text { No } & \text { ref. } & - & \text { ref. } & - & \text { ref. } & - & \text { ref. } & - \\ \text { Yes } & 1.24 & 0.326 & 1.37 & 0.099 & 1.82 & 0.045 & 1.32 & 0.225 \\ & (0.81 \text { to } & & (0.94 \text { to } & & (1.01 & \text { to } & (0.84 & \text { to } \\ & 1.91) & & 1.98) & & 3.29) & & 2.07)\end{array}$

T stage

$\begin{array}{lllllllll}1-2 & \text { ref. } & - & \text { ref. } & - & \text { ref. } & - & \text { ref. } & - \\ 3-4 & 0.94 & 0.808 & 1.21 & 0.419 & 1.42 & 0.365 & 1.16 & 0.607 \\ & (0.57 \text { to } & & (0.76 \text { to } & & (0.66 & & (0.67 & \text { to } \\ & 1.54) & & 1.91) & & \text { to } & 3.06) & & 2.01)\end{array}$




\begin{tabular}{|c|c|c|c|c|c|c|c|c|}
\hline $0-1$ & ref. & - & ref. & - & ref. & - & ref. & - \\
\hline $2-3$ & $\begin{array}{l}2.02 \\
(1.15 \text { to } \\
3.54)\end{array}$ & 0.014 & $\begin{array}{l}2.02 \\
(1.24 \text { to } \\
3.27)\end{array}$ & 0.004 & $\begin{array}{l}1.31 \\
(0.66 \\
\text { to } \\
2.59)\end{array}$ & 0.436 & $\begin{array}{l}2.60 \\
(1.37 \\
\text { to } \\
4.92)\end{array}$ & 0.003 \\
\hline
\end{tabular}

\section{Clinical stage}

\begin{tabular}{lllllllll}
3 & ref. & - & ref. & - & ref. & - & ref. & - \\
\hline $4 a$ & 2.57 & $<0.001$ & 2.41 & $<0.001$ & 1.84 & 0.042 & 2.87 & $<0.001$ \\
& $(1.66$ to & & $(1.66$ to & & $(1.02$ & & $(1.82$ & \\
& $3.97)$ & & $3.49)$ & & to & & to & \\
& & & & & & $3.29)$ & & $4.54)$
\end{tabular}

Induction

chemotherapy

$\begin{array}{lllllllll}\text { TP } & \text { ref. } & - & \text { ref. } & - & \text { ref. } & - & \text { ref. } & - \\ \text { TPF } & 0.85 & 0.541 & 1.03 & 0.897 & 0.78 & 0.498 & 1.08 & 0.765 \\ & (0.49 \text { to } & & (0.67 \text { to } & & (0.37 & & (0.64 & \\ & 1.45) & & 1.59) & & \text { to } & & \text { to } & \\ & & & & & & 1.61) & & 1.82)\end{array}$

Concurrent

chemotherapy

\begin{tabular}{lllllllll}
\hline TP & ref. & - & ref. & - & ref. & - & ref. & - \\
\hline$P$ & 1.72 & 0.041 & 1.58 & 0.042 & 1.03 & 0.936 & 1.77 & 0.043 \\
& $(1.02$ to & & $(1.02$ to & & $(0.55$ & & $(1.02$ & \\
& $2.88)$ & & $2.45)$ & & to & & to & \\
& & & & & & $1.93)$ & & $3.07)$
\end{tabular}

CT drug base

on DOC/TXT

$\begin{array}{lllllllll}\text { DOC } & \text { ref. } & - & \text { ref. } & - & \text { ref. } & - & \text { ref. } & - \\ \text { TXT } & 1.81 & 0.058 & 1.67 & 0.083 & 0.52 & 0.370 & 2.22 & 0.015 \\ & (0.98 \text { to } & & (0.94 \text { to } & & (0.13 & & (1.17 & \\ & 3.35) & & 2.98) & & \text { to } & & \text { to } & \\ & & & & & & 2.16) & & 4.22) \\ \end{array}$

CT drug base on DDP/NDP

$\begin{array}{lllllllll}\text { DDP } & \text { ref. } & - & \text { ref. } & - & \text { ref. } & - & \text { ref. } & - \\ \text { NDP } & 1.24 & 0.390 & 1.14 & 0.549 & 1.39 & 0.364 & 1.13 & 0.645 \\ & (0.76 \text { to } & & (0.74 \text { to } & & (0.69 & & (0.67 & \\ & 2.05) & & 1.75) & & \text { to } & & \text { to } & \\ & & & & & & 2.80) & & 1.90) \\ \end{array}$

Platinum dose of CCRT, $\mathrm{mg} / \mathrm{m} 2$ 


$\begin{array}{lllllllll}<150 & \text { ref. } & - & \text { ref. } & - & \text { ref. } & - & \text { ref. } & - \\ \geq 150 & 0.65 & 0.049 & 0.72 & 0.089 & 0.96 & 0.897 & 0.72 & 0.151 \\ & (0.42 \text { to } & & (0.50 \text { to } & & (0.54 & & (0.46 & \\ & 1.00) & & 1.05) & & \text { to } & & \text { to } & \\ & & & & & & 1.73) & & 1.13)\end{array}$

Table 3. Multivariate Cox proportional-hazard regression of independent variables to OS, PFS, LRRFS, and DMFS 


\begin{tabular}{|c|c|c|c|c|c|c|c|}
\hline & OS & & PFS & & LRRF & & DMFS \\
\hline Parameters & $\begin{array}{l}\mathrm{HR}(95 \% \\
\mathrm{Cl})\end{array}$ & $\mathrm{P}$ & $\begin{array}{l}\mathrm{HR}(95 \% \\
\mathrm{Cl})\end{array}$ & $P$ & $\begin{array}{l}\text { HR } \\
(95 \% \\
\mathrm{Cl})\end{array}$ & $P$ & $\begin{array}{l}\text { HR } \\
(95 \% \\
\mathrm{Cl})\end{array}$ \\
\hline
\end{tabular}

Age, year

$\begin{array}{lll}<46 & \text { ref. } & - \\ \geq 46 & 1.67 & 0.024 \\ & (1.07 \text { to } & \\ & 2.60)\end{array}$

\begin{tabular}{lll} 
BMl, kg/m2 & 0.033 \\
\hline$<18$ & ref. & - \\
\hline $18-24$ & 1.18 & 0.744 \\
& $(0.43$ & to \\
& $3.27)$ \\
\hline 24 & 0.53 & 0.267 \\
& $(0.17$ & to \\
& $1.63)$ &
\end{tabular}

Smoking

No

ref.

$1.79 \quad 0.051$

Yes

(1.00 to

3.23)

$\mathrm{N}$ stage

$\begin{array}{lllll}0-1 & \text { ref. } & - & \text { ref. } & - \\ 2-3 & 2.45 & 0.003 & 2.20 & 0.002 \\ & (1.36 \text { to } & & (1.34 \text { to } & \\ & 4.41) & & 3.62) & \end{array}$

ref.

$3.33<0.001$

(1.71

to

6.45)

Clinical stage

\begin{tabular}{lllllllll}
3 & ref. & - & ref. & - & ref. & - & ref. & - \\
\hline $4 a$ & 2.65 & $<0.001$ & 2.48 & $<0.001$ & 1.80 & 0.048 & 2.85 & $<0.001$ \\
& $(1.71$ to & & $(1.71$ to & & $(1.01$ to & & $(1.79$ & to \\
& $4.10)$ & & $3.61)$ & & $3.24)$ & & $4.54)$
\end{tabular}

CT drug base on DOC/TXT

DOC

ref.

ref.

ref. 
TXT

2.47

(1.31 to

4.67)
0.005

2.40

(1.32 to

4.37)
0.004

$3.25<0.001$

(1.66 to

Table 4. Side effects

\begin{tabular}{ll} 
Parameters & N (\%) \\
\hline Hematology & \\
\hline \multicolumn{1}{c}{0} & $62(11.90 \%)$ \\
\hline $1-2$ & $310(59.50 \%)$ \\
\hline $3-4$ & $149(28.60 \%)$ \\
\hline Radiation-induced oral mucositis & \\
\hline 0 & $1(0.18 \%)$ \\
\hline $1-2$ & $401(73.85 \%)$ \\
\hline $3-4$ & $141(25.97 \%)$ \\
\hline Gastrointestinal reaction & \\
\hline 0 & $30(5.52 \%)$ \\
\hline $1-2$ & $479(88.21 \%)$ \\
\hline $3-4$ & $34(6.26 \%)$ \\
\hline Liver function & \\
\hline 0 & $260(62.35 \%)$ \\
\hline $1-2$ & $151(36.21 \%)$ \\
\hline $3-4$ & $6(1.44 \%)$ \\
\hline Renal function & $369(88.49 \%)$ \\
\hline 0 & $48(11.51 \%)$ \\
\hline $1-2$ &
\end{tabular}

Figures 

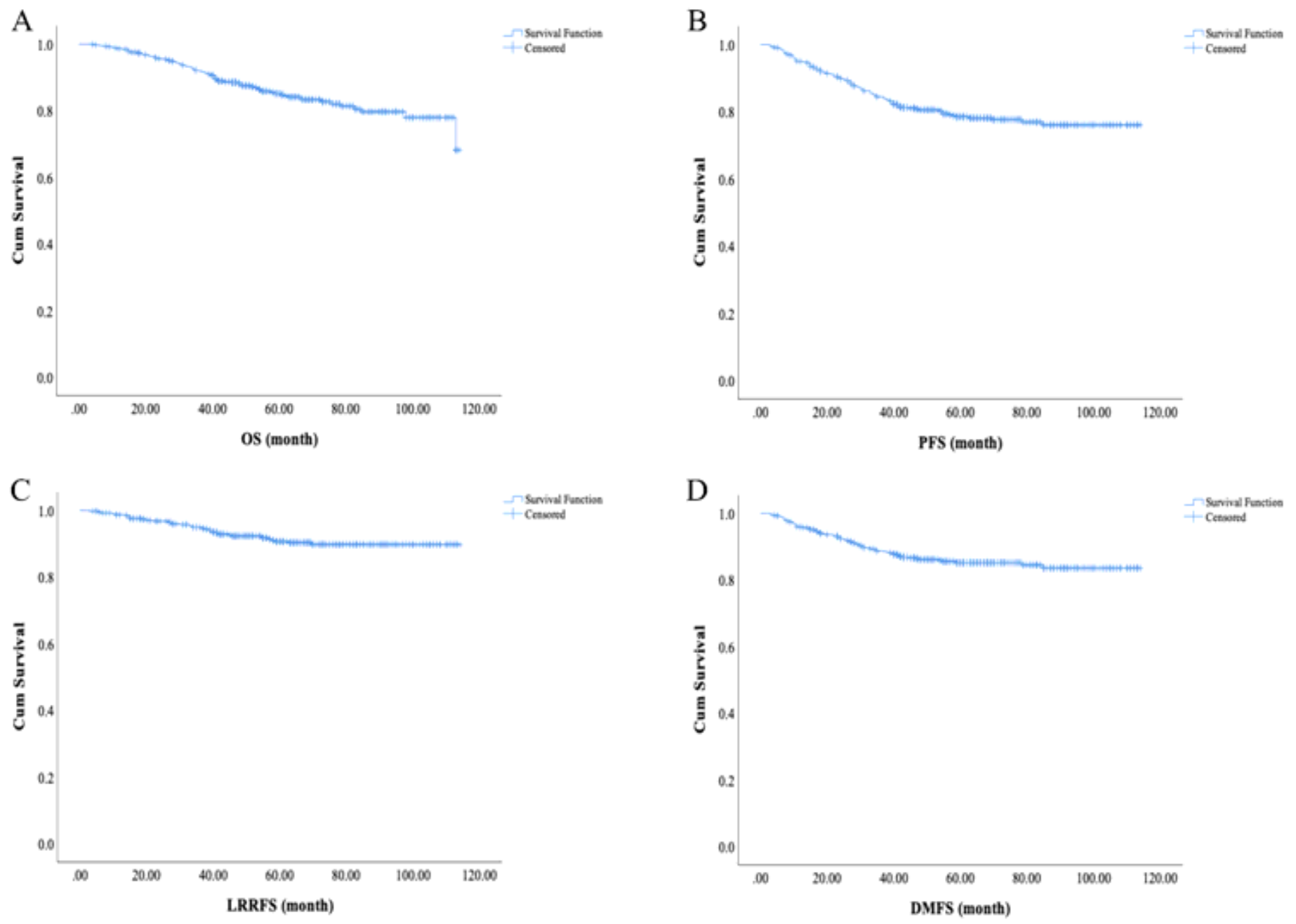

Figure 1

The Kaplan-Meier survival results of OS (A), PFS (B), LRRFS (C), and DMFS (D) in all patients. 

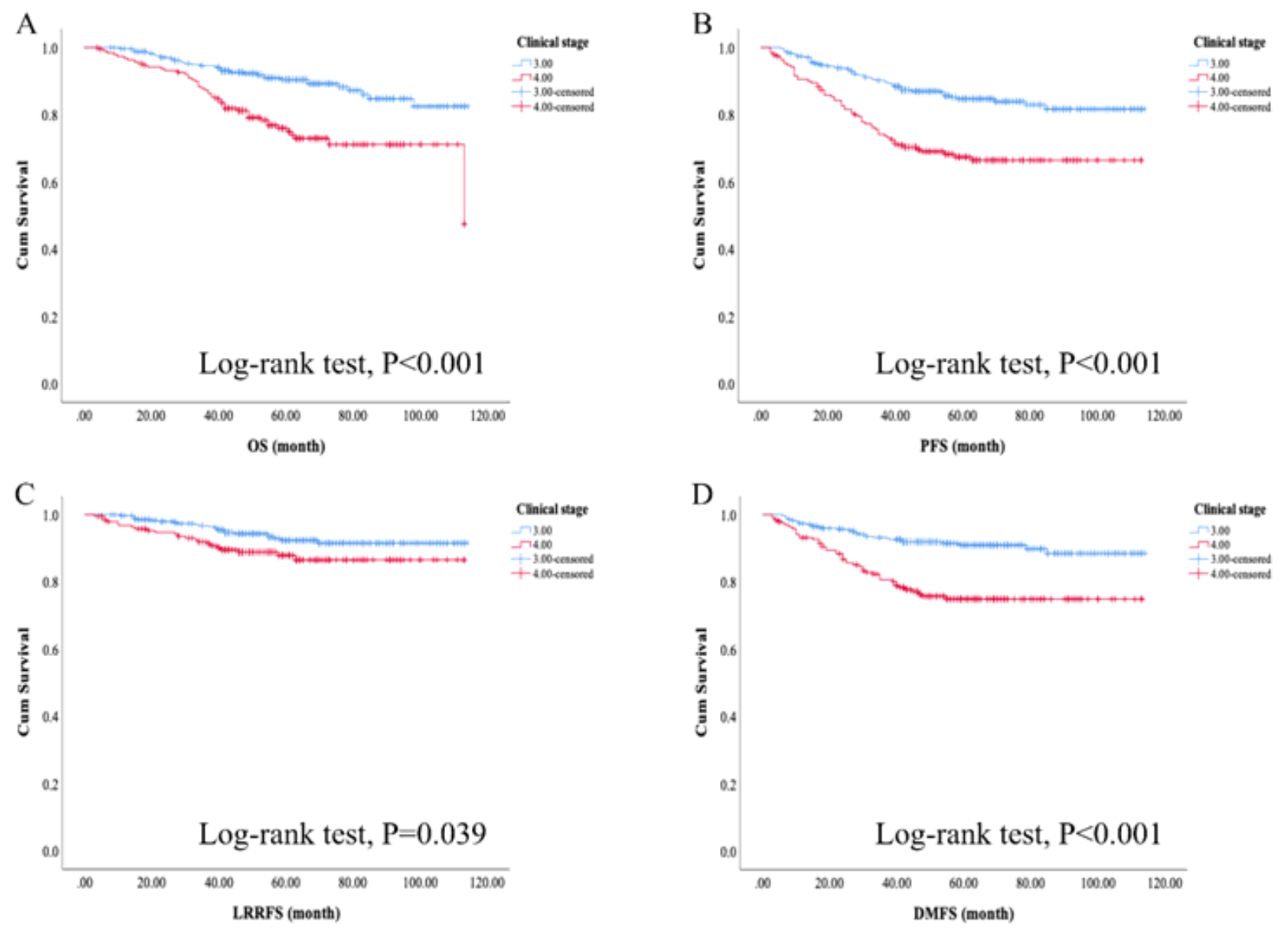

Figure 2

The Kaplan-Meier survival results of clinical stage to OS (A), PFS (B), LRRFS (C), and DMFS (D). 